Theories \& Applications, the International Edition

Printed Version : (ISSN 2090-5262)

Online Version : (ISSN 2090-5270)

July 2014, Volume 4, No. 2 Pages (32 - 38)

\title{
The Effect of a Specific Short-Term Training Program on Correcting the Curvature Angle of Kyphosis in Female Young Gymnasts.
}

\section{Wafaa Gaber}

Lecturer in the Department of Health Subject, the Faculty of Physical Education "for Women" - Alexandria University - Egypt.

\begin{abstract}
As the trend now is increasing towards using sports exercises and physiotherapy instead of medical and surgical interference, this study aims for comparing the effect of 8 weeks specific short-term training program in water and land on the angle of kyphosis and some pulmonary indices in kyphotic young female gymnasts that takes place as a result of their specific performance. Thirty female gymnasts with increased normal thoracic kyphosis (kyphosis $\geq 40$ degrees, Age: 13.2 +/3.79 years, weight: $34.84 \pm 6.44 \mathrm{~kg}$, height: $142.9 \pm 11.26)$ were selected and randomly divided in two groups $(n=15):$ group (1) (corrective exercise in water) and group (2) (corrective exercise in land). The angle of kyphosis was measured by using a flexible ruler as a non-invasive and reliable method. Pulmonary indices such as: force vital capacity (FVC), forced expiratory volume in one second (FEVI) and maximal voluntary ventilation (MVV) measured by digital spirometer. The repeated measurement test was used to compare the differences between the pre and post-tests in both groups. Statistical analysis was performed using SPSS statistical software version 18 and the significance level was set on 0.05. In land group and in water group the degree of kyphosis, FVC, FEV1 and MVV improved significantly ( $\leq \leq 0.05)$. Finally, between two groups were not observed any significant difference in degree of kyphosis and pulmonary indices (FVC, FEVI, MVV) $(p \geq 0 / 05)$. According to the results; we can suggest using in water and in land physical exercises for correction of the curvature angle of kyphosis in female young gymnasts and applying further studies on different samples and using different types of exercise protocols to avoid surgical interference and increase self-esteem for those young members of society.
\end{abstract}

Key words: gymnasts, kyphosis, aquatic exercise therapy, spirometer, corrective exercise.

Introduction

$\mathrm{K}$ yphosis (from Greek - kyphos, a hump), also called round back or Kelso's hunchback, is a condition of over-curvature of the thoracic vertebrae (upper back). It can be either the result of degenerative diseases (such as arthritis), developmental problems (the most common example being Scheuermann's disease), osteoporosis with compression fractures of the vertebrae, or trauma. In the sense of a deformity, it is the pathological curving of the spine, where parts of the spinal column lose some or all of their lordosis profile. This causes a bowing of the back, seen as a slouching posture. While most cases of kyphosis are mild and only require routine monitoring, serious cases can be debilitating. As mentioned by Hassanin (2003) that high degrees of kyphosis can cause severe pain and discomfort, breathing and digestion difficulties, cardiovascular irregularities, neurological compromise and, in the more severe cases, significantly shortened life-spans. These types of high end curves typically do not respond well to conservative treatment, and almost always warrant spinal fusion surgery, which can successfully restore the body's natural degree of curvature.

Fig (1)

The difference between kyphotic and normal spine curvatures
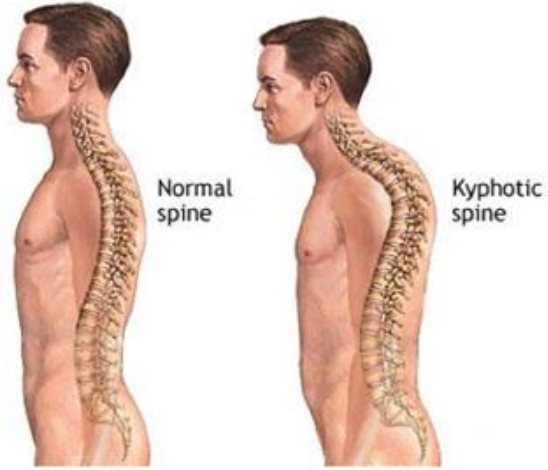
In the majority of the patients, thoracic kyphosis usually is painless and flexible. When symptomatic, pain may be aggravated by physical exertion; however, persistent pain may have other causes. Back pain is more common in high competitive athletes, and in athletes with high demands on their back, such as wrestlers and gymnasts. Panayoitis et al (2008) stated that deformity and cosmetic appearance are also common in all patients, especially the young and the adolescents. Gymnastics as an extension sport characterized with hyperextension exercises that are associated with jumping, marked stress and functional overuse of the back will definitely lead to a kyphotic back.

Brekaa \& Roushdy (1997) stated that any change or deviation of the spine from the normal position will lead to the malfunction of body. Abdelreheem (2011) stated that since the spine has a very important role in creating the movement and holding the body framework, and bears a significant mechanical internal and external pressure for performing daily activities, the lack of prevention and correction of abnormalities related to it, can lead to the secondary ab VB normalities in other parts of body and impedes the individuals' mental health and endanger their physical health. Abnormalities of spine will disturb the physical condition and cause serious psychological disorders. Kyphosis, which is one of the of the spine abnormalities, leads to various symptoms including: the short stature, breathing difficulties, muscle inflammation or spasm, nerve problems, rounded shoulders, increased lumbar lordosis, shortening the anterior ligament, strained longitudinal ligament, and disorder in lung expansion. In this complication, the trapezoidal middle, lower trapezoidal, and Erector spine muscles are extended and in contrast the intercostal, large chest, and shoulder adductors muscles are shortened.

In 2005, Arshadi found a significant relationship between the back extensor muscle strength and the level of kyphosis and lordosis in a research study entitled "Evaluating the relationship between the back extensor muscle strength and the flexibility of spine with the level of kyphosis and lordosis. Regression analysis showed that the back extensor muscle strength was the best predictor of kyphosis and lordosis level. Researchers indicated that the effect of therapeutic actions on the energy consumption and some related parameters in14-10 year-old kyphotic girls that the therapeutic exercises decreased the energy consumption, breathing rate, blood pressure, and heart rate, and increased the chest extension, and flexibility of spine, but the range of shoulder motion did not change.

Natural alignment of spinal column depends on the function of its muscle, bone and joint structure. This weakness of spinal column extensor muscles can cause static, dynamic and stature unbalance in persons that called faulty posture. It is caused unfavorable status on mental, social and physiological Function of a person (Nitzschke \& Hildenbrand, 1990; Peters, 1987). Physiological disorders caused by unfavorable physical status are serious. Increasing back curve of thoracic area that recognized as kyphosis has the unfavorable result of the respiratory system by the shortness and the inflexibility of chest muscles and also the weakness of expiratory muscles (Taheritizabi et al.,2012). Because shortening weakness in chest muscles of aspiration cause to decreasing ribcage volume, then decreasing lung volume (Rajabi et al., 2008). Therefore it seems effective to improve kyphosis and pulmonary volumes by reformation of muscle-skeletal structure of ribcage and spinal column, by corrective protocols of strengthening back muscles of ribcage and stretching of muscle chest. In the field of rehabilitation, physical attributes of water have many advantages like muscular relaxation, decreasing sensitivity to pain, decreasing cramp, flowing and comfort of joint, increasing muscular power and endurance when a person is very weak. Practice in water in comparison to practice in land is a certain method to achieve mental calmness and has many advantages to quality of life.

Many people who have unsuitable organs and look and also have congenital means of physical weakness of illness or even over weight and weak person don't like to do social activities or even if they want, they can't do any exercise whereas aquatic exercise solved this problem (Sato et al., 2007; Tsukahara et al., 1994; Wenger et al., 1984). The purpose of this research is comparing the effect of corrective particular exercise in water and land on changes of kyphosis and some respiratory indices in male students. To find answers to this question, which exercises methods (water or land exercise), has more affection on kyphosis reformation and pulmonary indices? Pulmonary volumes gathered by GAGER spirometer system in following conditions, each object is sitting on lab chair and getting sensor of the system in his hand then cross expiration and inhalation through a replaceable tube. The object should get the tube by lips and uses a particular set for black nose to not go out the air. Each subject done each test three times and the best records were gathered and registered at MVV, FEV1 and FVC. Exercising protocol on land:

1. Warm up: inclusive walking and running slowly about 5 or $10 \mathrm{~min}$.

2. Particular stretching exercises: all static stretching actions were done by him or his assistant, the purpose of static stretching was changing the length of shortened muscles to its natural length in the front chest area and its duration was 20 or 25 min. The period of each stretching action was 10 or 15 second and in $15^{\text {th }}$ sets. In the exercise pain threshold was important.

3. Particular strengthening exercises: It was done single and two times. Exercises were static or isometric and dynamic (isotonic). The main purpose of the exercises was the retrieval power of weakened back muscles, its duration was 20 or 25 minutes, the duration for each static strengthening action was 10 or 15 seconds in 5th or 10th sets.

4. Cool down period: running slowly and stretching action was done at the end about 5 or $10 \mathrm{~min}$. 
Exercising protocol in water: all of exercises in land were done in water. But the exercises were proportionate to aqueous area. For example making warm up and cool down exercises include different walking was done in the pool. ANOVA repeated measure test was used to compare the differences between groups in pre \& post-test. All statistical analyses were performed using SPSS statistical software version 18 and the significance level was set at 0.05 .

\section{Materials and Methods}

\section{Research Methodology}

Statistical Sample and the way for selecting them Statistical population in this research included 30 female students in prepatory and secondary schools in Alexandria who had postural kyphosis and were selected on target. (Age: $13.2+$ 3.79 years, weight: $34.84 \pm 6.44 \mathrm{~kg}$, height: $142.9 \pm 11.26$ )

\section{Data Collection Method}

The angle of kyphosis was measured by using a flexible ruler as a non-invasive and reliable method as proven by Rajabi (2008). Pulmonary indices such as: force vital capacity (FVC), forced expiratory volume in one second (FEV1) and maximal voluntary ventilation (MVV) measured by digital spirometer.
According to the research objectives, the specified program was planned for six weeks. Subjects participated in the program three sessions per week. 30 minutes of each exercise session was useful. In order to do these exercises, after five minutes general warming up, the subjects started strength exercises for back muscles and extension exercises for muscles in front of chest. And finally they cooled the muscles 4 minutes.

\section{Statistical method}

Collected data were analyzed using the SPSS software. Dependent t-test was used in order to compare the pre-and post-test program of specific water-land training exercises. The significant level was considered $\mathrm{p}<0.05$ for data analysis.

\section{Results}

As for kyphosis angle interactive effect $\mathrm{F}(1,28)=0.102$, $\mathrm{P} \geq 0.05$, isn't significant and group interior effect of the test $\mathrm{F}(1,28)=192.92, \mathrm{P} \geq 0.05$. So kyphosis angle increase significantly (Fig.2).

Figure (2)

Mean thoracic kyphosis angle (degree)

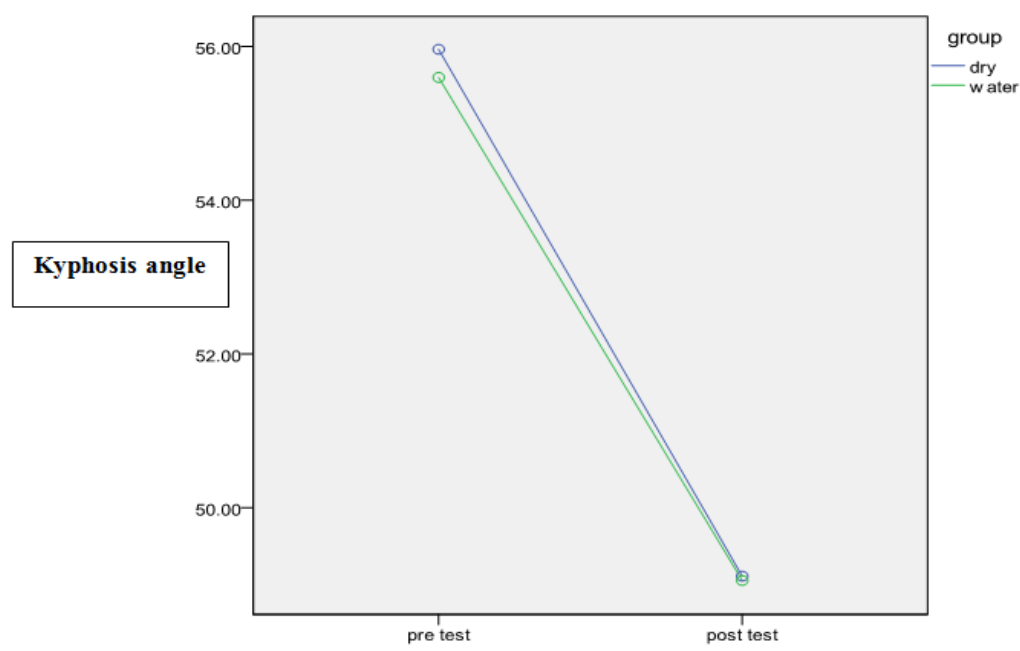

As for FEV1 interactive effect isn't significant, $\mathrm{F}(1,28)=0.28 / \mathrm{P} \geq 0.05$, and inter group test effect are significant, $F(1,28)=13.40 / \mathrm{P} \leq 0.01$, result $\mathrm{FEV} 1$ in two groups of aquatic and land increased so much over a period of particular corrective exercises (Fig.2). Also there aren't any significant differences between variations of kyphosis degree in two groups of aquatic and land $\mathrm{F}(1,28)$ $=0.001 / \mathrm{P} \geq 0.05$. 
Figure (3)

FEV1 values ( $\mathrm{mm})$ in groups before and after 8 weeks specific corrective exercise in water and land

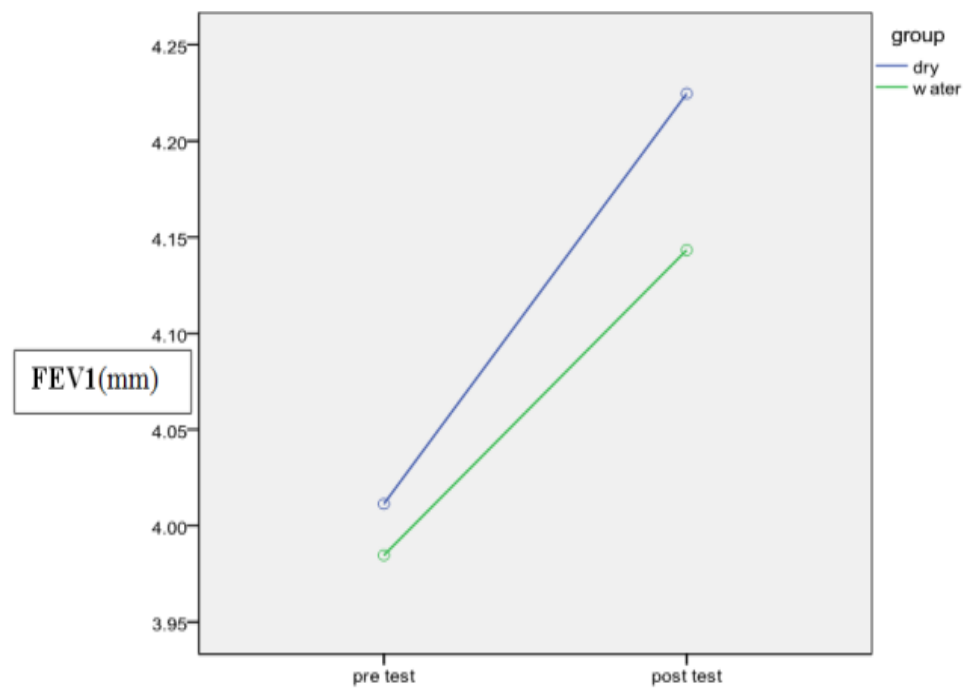

Finally, there is no significant difference in interactive effect $\mathrm{F}(1.28)=1.736, \mathrm{P} \geq 0.05$ and there is a significant difference in intergroup effect $\mathrm{F}(1.28)=23.381, \mathrm{P} \leq 0.001$, consequently FVC value increases significantly in both groups after 8

weeks specific corrective exercise in water and land (Fig. 3). There aren't any significant differences in

FVC between subjects in both groups $F(1.28)=0.001$, $\mathrm{P} \geq 0.05$.

Figure (4)

FVC values ( $\mathrm{mm})$ in groups before and after 8 weeks specific corrective exercise in water and land

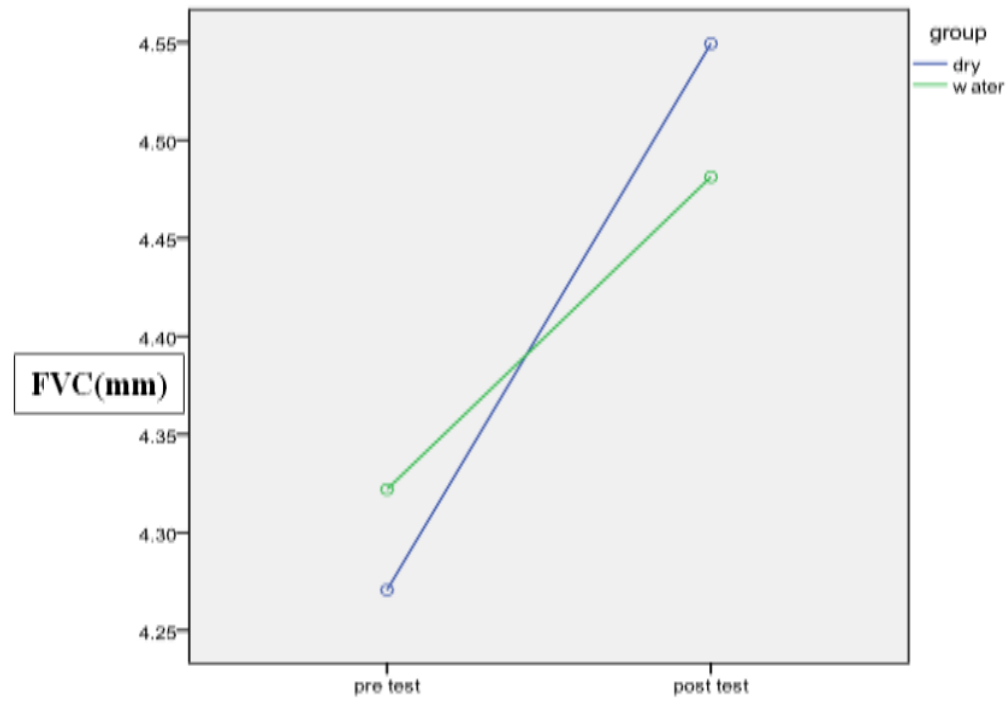

As for MVV the connected effect isn't significant in $\mathrm{F}$ land group is so much increased over a period of particular $(1.28)=0.129 / \mathrm{P} \geq 0.05$ and the inter group effect of the test $\mathrm{F}$ corrective exercises.

$(1.28)=19.55 / \mathrm{P} \leq 0.001$, results $\mathrm{MVV}$ in both aquatic and 
Figure (5)

MVV values (mm) in groups before and after 8 weeks specific corrective exercise in water and land

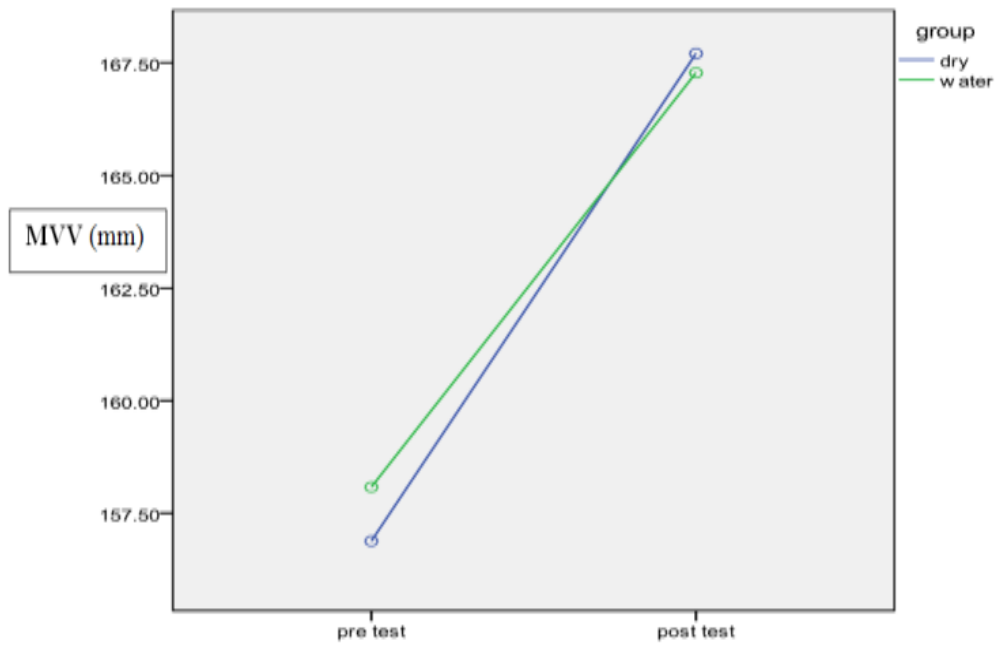

\section{Discussion and Conclusion}

According to the research findings, it has been indicated that the therapeutic exercise has a significant effect on all of the research variables. The research shows there is an important decrease in both kyphosis angle and important reformation in MVV, FEV1 and FVC after a particular corrective aquatic and land exercise in both groups (aquatic exercise group and land exercise group). Eun-Hee, Haley, Goodman and Fairweather show through corrective exercises to increase power of back extensor muscles, the kyphosis would decrease (Choi et al., 2005; Lynn, 2001; Hrysomallis \& Goodman, 2001; Fairweather \& Sidaway, 1993), moreover Mika, Sinaki, Briggs also show in their researches there is inverse correlation between power of back extensor muscles and level of kyphosis thus level of back disorders can be result of decrease power of back extensor muscles their results are same to our results (Mika et al., 2005; Sinaki et al., 1996; Sinaki et al., 2005; Briggs et al., 2004), unlike results of Sedlock and Egan are different, they declared that level of back extensor muscles power has slight effect on level of kyphosis (Eagan \& Sedlock, 2001; Kim et al., 2006).

Hildenbrand and colleagues performed an aquatic athletic exercise program in water on asthmatic patients for 12 weeks, they didn't observe any significant difference between pre-test and posttest in FEV1 and FVC that was different by the result of this research, but the sample of this research was asthmatic patients (Hildenbrand et

al., 2010). Anonymous research's results showed that aquatic athletic treatment lead to important increases in FEV \& MVV in 10 weeks in respiratory operation of MR patients in educable group, their results is similar to the current study (Can et al., 2005). The reason of decrease kyphosis angle in samples after a period of land \& aquatic corrective exercises is strengthened stretched muscles in the backside of the spinal column (back extensor muscles, through strengthening and endurance corrective exercises in water and land and also flexibility exercises in chest area using help and without help.

Samples in water (Flotage principle) and inland caused volubility of the spinal column and reform of shortened muscles too. Regarding to effect of particular corrective land $\&$ aquatic exercises on (MVV, FEV1, FVC) and important improvement of them, it's noticeable that probably decrease in kyphosis angle using corrective exercise caused not only increasing in pulmonary ribcage compliance and ventilation but also decreasing in aerial path resistance and lung tissue resistance, and lungs get more capacity so this can reform all pulmonary indices. On the other hand more over these factors, reinforce intercostal muscles and respiratory muscles impress pulmonary indicators (MVV, FEV1, FVC), probably important in all pulmonary MVV, FEV1, FVC is related to this factor by doing aerobic exercises in warm up and cool down exercises. Also hydrostatic pressure made by floating organs or body in water,

makes physiological response of the circulatory system. As hydrostatic pressure results leading blood from lower extremity to the trunk and body and cusses of stroke volume level, cardiac output and vo2max and then the pulmonary volume will increase. It should point that this research is one of few researches that studied the effect of aquatic exercise on kyphosis and pulmonary volumes. Nevertheless many recommendations of physiotherapist about using aquatic exercise there are few researches in this field. Therefore it's necessary to more researches about aquatic exercise on posture and disorders. Also in the research method of variance analysis with repeated measure shows there isn't important difference in two groups in each related variables. There isn't any difference between two groups, results show that the changes in each studied variable isn't related to particular exercise area, but both exercise area (water \& land) shows equal effect on this variable statically. 
Recommendation:

According to the results; the researcher can suggest using in water and in land physical exercises for correction of the curvature angle of kyphosis in female young gymnasts and applying further studies on different samples and using different types of exercise protocols to avoid surgical interference and increase self-esteem for those young members of society.

References

1. Abd Elreheem N. A.: Rehabilitation exercise for postural education. (2011), Dar elfekr - Jordan.

2. Breka'a M. G., Roushdi A.: The mechanism of spinal chord injuries (1997), Monshaet el-Maaref, Alexandria - Egypt

3. Briggs, A.M., Greig, A.M., Wark, J.D., Fazzalari, N.L., \& Bennell, K.L. (2004). A Review of anatomical and mechanical factors effecting vertebral integrity. Int J Med Sci, 1(3), 170-180.

4. Can, F., Ergun, N., Yilmaz, Y., Bayrak, C., Konukman, F., Agbuga, B. et al. (2005). Effect of a 10-week swimming program on the respiratory function of children with mental retardation. Research Quarterly for Exercise \& Sport, 76(1), A-27.

5. Choi, E.H., Hur, J.K., Yang, J.I., \& Park, D.S. (2005). Poster 98: The Effect of Thoracic Exercise Program on Thoracic Pain, Kyphosis, and Spinal Mobility. Archives of physical medicine and rehabilitation, 86(9), e23.

6. Eagan, M.S., \& Sedlock, D.A. (2001). Kyphosis in active and sedentary postmenopausal women. Med Sci Sports Exerc, 33(5), 688-695.

7. Fairweather, M.M., \& Sidaway, B. (1993). Ideokinetic imagery quarterly as a postural development technique. Res Q Exerc Sport, 64(4), 385-392.

8. Goodman c, Hrysomallis c, Journal of strength and condition research. 2001, 235-249.

9. Hassanin M. S, Ragheb M. A.: Perfect posture for all (2003), Dar elfekr elarabi, Cairo - Egypt.

10. Hildenbrand, K., Nordin, S., Freson, T.S., \& Becker, B.E. (2010). Development of an aquatic exercise training protocol for the asthmatic population. International Journal of Aquatic Research and Education, 4(3), 278-299.
11. Hrysomallis, C., \& Goodman, C. (2001). A Review of Resistance Exercise and posture realignment. J StrengthCond Res, 15(3), 385-390.

12. Kim, H.J., Chung, S., Kim, S., Shin, H., Lee, J., Kim, S., \& Song, M.Y. (2006). Influence of trunk muscle on lumbar lordosis and sacral angle. Eur Spine J, 15(4), 409-414.

13. Lynn, H. (2001). Exercise may ease kyphosis effect. Improving posture and muscle strength reduce curvature of spin. Journal of American College of Rheumatology.

14. Martha R.Hinman, EdD, PT, The Spine of Journal $2004,413-417$

15. Mika, A., Unnithan, V.B., \& Mika, P. (2005). Differences in thoracic kyphosis and in back muscle strength in women with bone loss due to osteoporosis. Spine, 30(2), 241-246.

16. Nitzschke, E., \& Hildenbrand, M. (1990). Epidemiology of kyphosis in school children. $Z$ Orthop Ihre Grenzgeb, 128(5), 477-481.

17. Panayiotis J. Papagelopoulos, MD, DSc; Andreas F. Mavrogenis, MD; Olga D. Savvidou, MD; Evanthia A. Mitsiokapa; George G. Themistocleous, MD; Panayotis N. Soucacos, MD, FACS: Orthopedics Current Concepts in Scheuermann's Kyphosis. January 2008 Volume $31 \cdot$ Issue 1

18. Peters, S. (1987). Electrocardiography changes in scoliosis and kyphosis deformities of the thorax. 125(1), 14-18.

19. R. Rajabi, F. Seidi and F. Mohamadi: Which Method Is Accurate When Using the Flexible Ruler to Measure the Lumbar Curvature Angle? Deep Pint or mid Point of Arch? World Applied Sciences Journal 4 (6): 849-852, 2008

20. Rajabi, R., Seidi, F., \& Mohamadi, F. (2008). Which method is accurate when using the flexible ruler to measure the lumbar curvature angle? Deep pint or mid point of arch? World Applied Sciences Journal, 4(6), 849-852.

21. Sato, D., Kaneda, K., Wakabayashi, H., \& Nomura, T. (2007). The water exercise improves health-releated quality of life of frail elderly people at day service facility. Qual Life Res, 16(10), 1577-1585. 
22. Sinaki, M., Brey, R.H., Hughes, C.A., Larson, D.R., \& Kaufman, K.R. (2005). Balance disorder and increased risk of fall in osteoporosis and kyphosis. Significance of kyphotic posture and muscle strength. Osteoporos Int, 16(8), 10041010.

23. Sinaki, M., Itoi, E., Rogers, J.W., Bergstralh, E.J., \& Wahner, H.W. (1996). Correlation of back extensor strength with thoracic kyphosis and lumbar lordosis in estrogen-deficient women. Am J Phys Med Rehabil,75(5), 370-374.

24. Taheritizabi, A., Mahdavinejad, R., Azizi, A., Jafarnejadgero, T., \& Sanjari, M. (2012). Investigation of sagittal curves of spinal column and establishing the norm of thoracic kyphosis and lumbar lordosis. World Journalof Sport Sciences. 6(1), 80-83.

25. Tsukahara, N., Toda, A., Goto, J., \& Ezawa, I. (1994). Cross-sectional and longitudinal student on the effect of water exercise in controlling bone loss in Japanese postmenopausal woman. J Nutr Sci Vitaminol, 40(1), 37-47.

26. Vaughna Daniel W, Brown Eugene W, Journal of Back and Musculoskeletal Rehabilitation 20, 2007, 155-165

27. Wenger, N.K., Mattson, M.E., Furberg, C.D., \& Elinson, J. (1984). Assessment of quality of life in clinical trials of cardiovascular therapies. Am J Cardiol, 54(7), 908-913.q 